\title{
Design and Simulation of Spectrum Access and Power Management Protocol for Dynamic Access Networks
}

\author{
Ala'eddin A. Masadeh ${ }^{1}$, Haythem Bany Salameh ${ }^{2,3}$, Ahmad Abu-El-Haija ${ }^{4}$ \\ ${ }^{1}$ Al-Balqa Applied University, Al-Salt, Jordan \\ ${ }^{2} \mathrm{Al}$ Ain University, Al Ain, UAE \\ ${ }^{3}$ Yarmouk University, Irbid, Jordan \\ ${ }^{4}$ Jordan University of Science and Tech., Irbid, Jordan
}

\begin{abstract}
This work investigates the problem of managing the transmission power and assigning channels for multi-channel single-radio Cognitive Radio Ad-Hoc Networks (CRAHNs). The considered network consists of M primary users and $N$ secondary users, where the secondary users can use the licensed channels opportunistically when they are not utilized by the primary users. The secondary users have the capability of sensing the licensed channels and determine their occupation status. They are also able to control their transmission power such that the transmitted data can be received with high quality-ofservice with the lowest possible transmission power, and minimum interference among the secondary users. This also contributes in increasing the frequency spatial reuse of the licensed channels by the secondary users, when the channels are unoccupied, which increases the network throughput. This work proposes a channel assignment algorithm aims at assigning the unoccupied licensed channels among secondary users efficiently, and a transmission power control aims at tuning the transmission power used by the secondary users to maximize the network throughput. The results show an enhancement achieved by the proposed protocol when it is integrated to the considered network, which is seen through increasing the network throughput and decreasing in the access delay. In this context, the Network Simulator 2 (NS2) was used to verify our proposed protocol, which indicates a significant enhancement in network performance.
\end{abstract}

Keywords: Cognitive radio network, primary user, secondary user, licensed spectrum, unlicensed spectrum, medium access control.

Received January 28, 2020; accepted June 9, 2020

https://doi.org/10.34028/iajit/17/4A/2

\section{Introduction}

Cognitive Radio Ad-Hoc Networks (CRAHNs) has been one of the attractive technologies, where many attempts have been proposed to develop these networks due to their attractive properties. These properties can be summarized by their ability to adjust their radio operating parameters dynamically and autonomously to modify the network operation. Cognitive Radio (CR) is distinguished from other conventional radios in two main features, which are the cognitive capability and reconfiguration $[2,3]$. The cognitive capability is defined as the capability of sensing and collecting information from the surrounding environment, such as frequency, power, and bandwidth. While the reconfiguration is known as the capability of adapting the operational parameters based on the collected information to optimize the network performance.

Due to spectrum sharing property, a Cognitive Radio Network (CRN) is an interference-intensive environment. Therefore, introducing mechanisms avoiding interference among CR users (both primary users (PUs) as well as Secondary Users (SUs)) is an important element for deploying this type of networks.
For any wireless network, the effect of the noise and interference differs from one network to another depending on the amount and type of the interference and the values of the transmission power used by the users in a network [18]. One of most important types of interference that should be considered in this type of networks is the self-interference. It is generated by the users themselves within a network and defined as the interference resulting from non-optimal positioning or transmitting power of the users within the network. Self-interference is an unwanted signal being received by non-intended users, which results in receiving data incorrectly. The main reason of this type of interference results from using the maximum transmission power used for transmitting data [18]. Optimizing the transmission power $[14,16]$ in a network contributes in reducing the self-interference, which maximizes the network throughput, and reduces the overall consumed energy resulting in increasing in the users' battery life. In this work, integrating the TPC to CRAHNs is proposed. Using this mechanism, it is possible to provide a network with the capability of saving power and coping with the scarcity of available spectrum, which are main features for any wireless network. In 
this work, the Cognitive Radio Cognitive Network $(\mathrm{CRCN})$ simulator was used. It is software-based network simulator that works in network simulator (NS2) environment. This simulator supports multi-channel single/multi-radio technology. Here, topology and traffic pattern files are generated using NS-2, where each user is equipped with a single radio multiple channel physical (PHY) layer [12]. The rest of this paper is organized as follows. In section 2, a brief overview about related work is presented. In section 3, the system model is described. The implementation of a multi-channel single radio power controlled MAC protocol for CRAHN in NS-2 is discussed in section 4 . The performance of the proposed MAC protocol (PC-CRMAC) is discussed in section 5 by presenting some simulation results. Finally, the conclusion is presented in section 6 .

\section{Related Work}

The problem of power control has been addressed by different schemes and protocols with various perspectives. Several TPC protocols have been introduced and studied, which can be classified into many types depending on several parameters. One of these classifications is to classify these protocols based on protocol's goal, which divides these protocols into two main types. The first one $[9,13]$ gets more attention on power saving more than throughput, whereas the second type $[15,17]$ makes the throughput as the main interest without taking into account the resources consumption and the system complexity in some approaches. In [25], a multi-channel multi radio MAC protocol with TPC was proposed, where the users have two half duplex transceivers. The first transceiver is used for control packets and the second one for data packets. Control packets are transmitted with maximum transmission power, while the data packets are transmitted at a controlled level with minimum needed power for encoding the received signal correctly. However, this is unreliable due to several reasons. One of these reasons is that initiating any new communications link will produce further interference, which reduces the throughput and adversely affects the performance of ongoing connections [16, 17]. MAC protocols can also be designed in a way that allows concurrent interference-limited transmissions [23], such as Power Controlled Multiple Access (PCMA), PowerControlled Dual Channel (PCDC), and Dual Busy-Tone Multiple Access (DBTMA). PCDC protocol was developed in [17]. This protocol uses two separate channels (the first one for control packets while the other for data packets). Here, for any transmission, a user should consider any possible future transmission to reduce the probability of collision. Computing the interference margin is the first step in this protocol, which is used to allow new users to transmit their data if their transmission power levels do not affect negatively on the existing transmissions. It is worth mentioning that this protocol uses RTS/CTS power levels to compute the interference margin, which aims at maximizing the number of concurrent connections in a neighbourhood. Channel assignment algorithms for CRNs are proposed in $[4,5]$. Here, CRNs allow SUs to utilize the licensed channels opportunistically, which requires channel assignment protocols that assign channels from the licensed and unlicensed bands to SUs in a way that does not degrade the performance of the overall network. Interference in CRNs has been investigated and handled by various researchers from different perspectives. Rabbachin et al., [19] proposed a new statistical model for aggregate interference for CRNs, where they derived the characteristic function and the cumulate of interference for CRN at the PUs. Here, truncated-stable distributions are used to obtain the statistical model for the cognitive network interference, which is used for capturing the statistical behavior of the CRN interference with different scenarios. In [11], an Adaptive MAC (AMAC) design for CRNs was designed. This protocol was designed to dynamically select protocol among the available MAC protocols according to changes on traffic or topology scenarios. Using AMAC, two commonly MAC protocols in wireless networks are used, which are CSMA and TDMA. CSMA is used for supporting short packet transmission with light to medium traffic, while TDMA is used in cases where the traffic scenarios are heavy. This protocol had come where most of research contributions considered throughput improvement, interference limitation, and power control [10, 19, 22] in the physical layer level of CRCN based on Dynamic Spectrum Access (DSA) technology. In this work, a TPC protocol is integrated to CRAHNs to permit multiple interference-limited concurrent transmissions. This has a great opportunity to enhance the performance of CRAHNs, where Network Simulator 2 (NS2) is used to verify the proposed theoretical findings.

\section{System Model}

In this work, a new TPC protocol as well as new channel assignment algorithm are integrated to CRAHNs. The proposed protocol that is called Power Controlled-Cognitive Radio MAC (PC-CRMAC) aims to reduce interference and increase the frequency spatial reuse in order to enhance the throughput performance. The main properties of this proposed protocol can be summarized as follows. Firstly, the power control algorithm is a per-packet algorithm. Secondly, this protocol does not introduce any new control packets other than RTS and CTS before DATA transmission, which reduces the complexity and cost of the system. Finally, this protocol uses single radio, which also reduces the cost and complexity of system. In the subsequent sections, the protocol will be explained in detail. 


\subsection{Propagation Model}

In wireless networks, the received power level can be computed based on different power propagation models to indicate the effect of the wireless channel on the transmitted signal. Here, in order to give more reliable results in case of long distances in line of sight path, Two-ray ground model is used, because this model takes into account both the direct path as well as the reflected path from the ground. According to the Two-ray ground model, the received power at the receiver is given by:

$$
P_{r}=P_{t}(K)^{2}\left(1 / d^{4}\right) g_{t} g_{r}
$$

Where $P_{t}$ and $P_{r}$ are transmitted and received power respectively, $d$ is the distance between the transmitter and receiver, $g_{t}$ and $g_{r}$ are the gains of the transmitting and receiving antennas respectively, $K$ is a constant that is determined based on heights of receiving and transmitting antennas. So, the path gain of the Two-ray ground model for the link between node $i$ and node $j$ is given as:

$$
G_{i, j}=\frac{P_{r}}{P_{t}}=(K)^{2}\left(1 / d^{4}\right) g_{t} g_{r}
$$

From the above equations, it can be concluded that the received power in the Two-ray ground model does not depend on the operational frequency of the signal. This is in contrast to the Free-space model, where the transmitted power decay is proportional to $\left(1 / d^{4}\right)$.

\subsection{Power Control Algorithm}

\section{A Table Maintained by Each Node}

Each node in this protocol has an information table that keeps important information about neighboring nodes, which are: MAC address of neighboring nodes, minimum power (denoted by $P_{\min }$, the minimum transmission power required for successful transmission when it does not suffer from any interference), interference tolerance (denoted by Itol), transmitted power (denoted by $P_{t}$, minimum power added to the tolerance), and channelindex, , which is the index (number) of the used channel from a predefined-channel list. Each time a node overhears from one of its neighbors, it updates its own table.

\section{Overhearing CTS}

Once a receiver $b$ receives RTS from a transmitter $a$, the receiver can detect the received power $P_{r}^{a b}$, which is represented mathematically according to Two-Ray ground propagation model by:

$$
P_{r}^{a b}=P_{t}^{a b}(K)^{2}\left(1 / d_{a b}^{4}\right) g_{t} g_{r}
$$

If the sensitivity of the receiver is denoted by $R X_{t h}$, and the channel is assumed to be symmetric, then the minimum power required by the receiver $b$ for successful transmission from the transmitter a can be represented as:

$$
P_{\min }^{a b}=\left(P_{t}^{a b} \cdot R X_{t h}\right) / P_{r}^{a b}
$$

After this, the receiver should check its own table to find its active neighbors. These active neighbors can be known using Network Allocation Vector (NAV) that indicates the time in which the neighbors finish their ongoing transmissions. After checking active neighbors of the receiver $b$, the maximum average interference generated by the neighbors of the receiver $b$ is computed as:

$$
P_{\text {interf }}^{b}=\frac{P_{r}^{a b}-\operatorname{SINR} \cdot P_{\text {noise }}}{N \cdot(1+\beta) \cdot \operatorname{SINR}}
$$

Where SINR is the signal-to-interference and noise ratio required to provide a required data rate (this value here is constant), $N$ is the number of neighboring nodes of the receiver $b$, and $\beta$ is the interference caused by nodes out of the transmission range, which is about half of the Two-ray propagation model [20]. It is important to note that this information is added to CTS frame. After sending CTS, most of neighboring nodes of the receiver b will receive this CTS that is used to update their neighboring tables. Once receiving the CTS, the minimum received power by a neighbor $\mathrm{k}$ from node $\mathrm{b}$ can be given by:

$$
P_{\min }^{b k}=\left(P_{t}^{b k} \cdot R X_{t h}\right) / P_{r}^{b k}
$$

\section{Tuning the transmitted power}

Setting an appropriate value to interference tolerance is the final step in this protocol. This value is added to $P_{\min }$ in order to use this new given value as a transmitted power. However, it is important to know that this procedure will be applied for each new transmission between any two different nodes.

The interference tolerance can be given as:

$$
\text { Itol }=\frac{P_{r}}{S I N R}-P_{\text {interf }}
$$

And the transmitted power is given by:

$$
P_{t}=P_{\text {min }}+\text { Itol }
$$

\subsection{Channel Assignment}

In this work the channel assignment algorithm is designed at MAC level, where the decision is made through the interfaces. Here, a new field channelindex is added into packet header for two purposes: to inform intended receiver as well as its neighbors about the selected channel, and to differentiate the channels to achieve conflict free or reduce interference among nodes in a neighborhood. At a time, when a pair of nodes needs to communicate with each other, the transmitter chooses an appropriate channel based on collected interference information. After this, it informs the intended receiver and its neighbors with channel selected by its neighbors using RTS at maximum power. Once the receiver receives RTS, it calculates the minimum required power, and selects the appropriate channel for communication based on its table and information from the transmitter. After this, the 
receiver sends the selected channel with an appropriate value of transmission power for using these parameters (transmit power and selected channel) for transmitting DATA and ACK.

As an example, consider the predefined table of the licensed channels that can be used opportunistically by SUs as shown below, where the number of these channels is 3 . The network has 10 SUs, where alphabets from a to $\mathrm{j}$ are used to indicate SUs. However, in addition to the predefined table of available channels, another table is used which is the channel distribution table. This table is stored by each node to save information about channels used by node's neighbors.

Table 1. Predefined table for available channels.

\begin{tabular}{|c|c|}
\hline Channel number & Status \\
\hline 1 & Free \\
\hline 2 & Used by primary user \\
\hline 3 & Free \\
\hline
\end{tabular}

Based on the above table and its information, the channels that can be used by SUs are 1, and 3. Once the first receiver selects a channel from the available ones, it broadcasts the number of this channel to inform the intended receiver as well as it neighbors by its CTS. Depending on received CTS, any new pair of nodes that wants to communicate in the neighborhood can select a channel that has not been used by its neighbors if one or more of the available channels are free. If all channels are used by its neighbors, then it will select the least frequently used channel in order to minimize the interference, reduce the collision, and for balancing the channel utilization as much as possible.

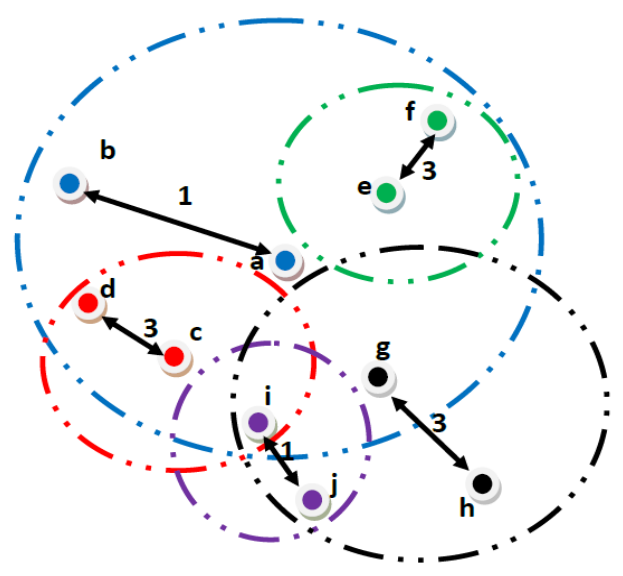

Figure 1. CRAHN supported with PC-MAC.

The below table is maintained by each node, which contains the communicating pairs and their selected channels. In this table, the first pair $(a, b)$ selects channel number 1 , where this number is saved by each node that hears the CTS. After this, when any new pair (such as $(\mathrm{c}, \mathrm{d}))$ needs to communicate, this pair negotiates to select a channel. Based on Tables 1 and 2, this pair will select channel number 3, because this channel is free. After this, any new pair that needs to communicate will use Tables 1 and 2 to determine and use the least frequently used channel.

Table 2. Table of channel distribution.

\begin{tabular}{|c|c|}
\hline Pair & Used channel \\
\hline $\mathrm{a}, \mathrm{b}$ & 1 \\
\hline $\mathrm{c}, \mathrm{d}$ & 3 \\
\hline $\mathrm{e}, \mathrm{f}$ & 3 \\
\hline $\mathrm{g}, \mathrm{h}$ & 3 \\
\hline $\mathrm{i}, \mathrm{j}$ & 1 \\
\hline
\end{tabular}

The effect of using the proposed protocol can be shown using Figure 1, which shows the network with the above assumptions, when TPC is used. Figure 2 shows the network with above assumptions without using TPC in CRAHN, where only two pairs can make communication. This is because of using maximum power in reserving channels and transmission using maximum power that cause collision at the receivers $f$, $d$, and $j$, which will prevent the completion of the communication process to these receivers. However, in case when the receiver is $h$, collision will not happen at this receiver, because it is not in the range of the transmitter a.

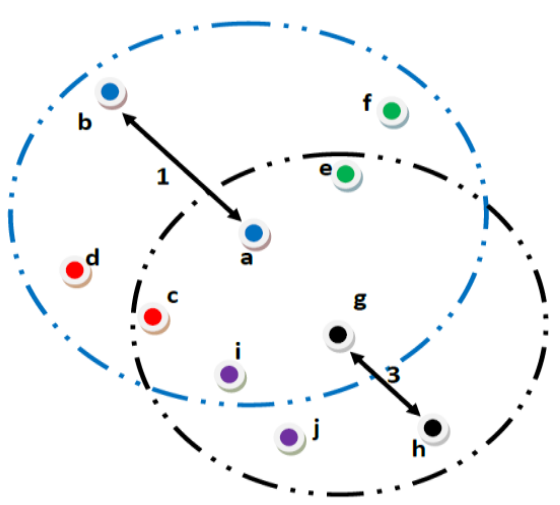

Figure 2. CRAHN without TPC protocol.

\section{The NS-2 Implementation of The Proposed MAC Protocol}

\subsection{Multi-Channel Support in NS-2}

This work mainly depends on CRCN simulator [7], which is a software based on NS-2. This simulator supports the functionalities for CR networking protocols (CR MAC, CR Routing, and CR PHY protocols). In this context, many functionalities have been used from this simulator such as multiradio/single-radio multi-channel support, interface to select radio/channel, and information needed during spectrum access.

Figure 3 shows the structure of single-radio multichannel structure for CRN in NS-2. As shown multiple channel copies are created for this structure. However, MAC protocols, TCL script, and mobile node in NS-2, which are in gray color, need modifications to support our proposed protocol. 


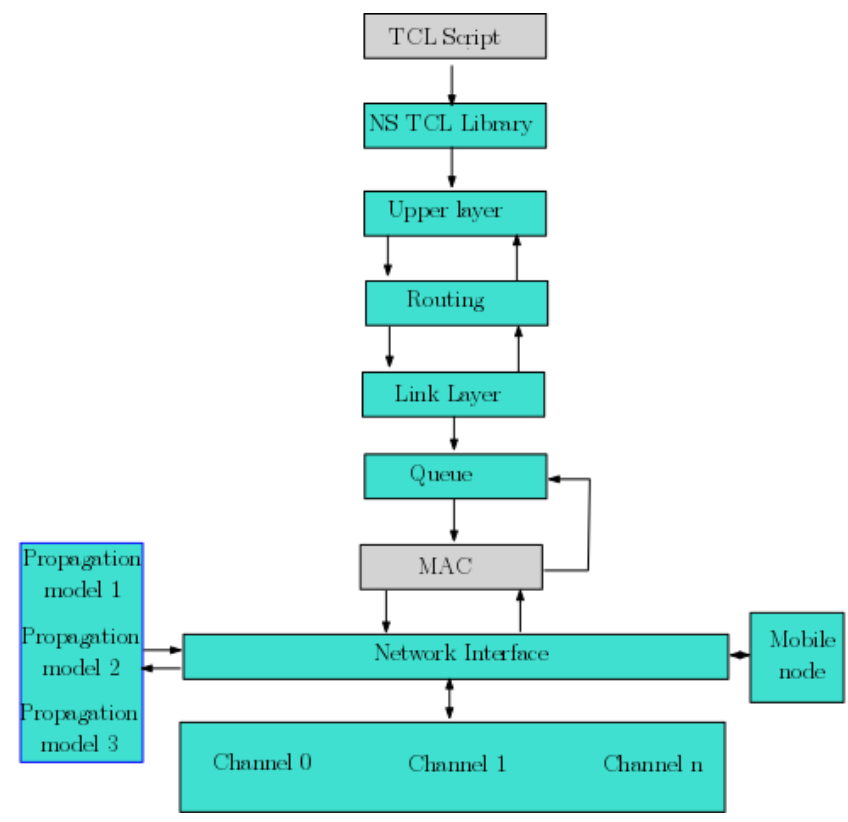

Figure 3. Design overview for single-radio multi-channel CRN [7].

For creating single-radio multi-channel, the channel decision from the $\mathrm{CR}$ routing is stored into the channelindex , which is a newly added variable by CRCN simulator in packet header file [packet.h], which is found in the "common" folder under NS-2 directory, where the lower layers have been modified to support that decision. However, this variable is set to zero by default, which means that the number of channels is one as a standard.

In TCL script level, it is important to be known that many statements should be added for supporting singleradio multi-channel. However, this can be summarized by adding the following 4 statements in TCL script, which is the main file that calls the required $\mathrm{C}++$ files. The first component is to set the number of channels:

set val(channum) $x$;\# where $x$ is the number of available channels

The second component is to add a new channel objects according to the interface number:

for $\{$ set i 0$\}\{\$ i<$ Sval(channum) $\}$ incr i\} \{

set chan_(\$i) [new \$val(chan)]; \#new channel objects )

The third component, which is used to configure multiple channel options:

\$ns_node-config -adhocRouting \$val(rp) \

hannelNum \$val(channum); \# configure channel number -channel \$chan_(0) ;\#configure the first channel object

The fourth component, which is the final one, and it is used to assign the channel objects to the channel array of the simulator: for \{ set i 0$\}\{\$ i<\$ v a l(n i)\}\{$ incr i\}

i

\$ns_add-channel \$i \$chan_(\$i); \# initialize the channel array with channel objects

\}

\subsection{Channel/Radio Decision}

Our work concentrates on implementing a contention based MAC, where each user can select a channel from a predefined channel list based on the distances among them. Each user must be aware of what channel being used by other users especially by the PUs. After selecting a channel by a user, the channel information is passed to PHY layer through the packet header to specify the appropriate transmission parameters, and to tell its neighboring users of the selected channel.

Channel decision in our MAC is implemented by defining a new variable in [mac-802_11.cc], which is the chandecision as follow:

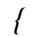

After determining the appropriate channel to be used for receiving from the designed CR MAC algorithm, the channel number should be stored in the predefined field by CRCN simulator, which is the channelindex_that is defined in the [packet.h] file as follow:

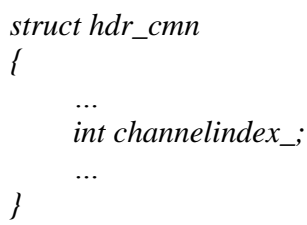

Note that channelindex_ field carries the information about the selected channel from CR routing, and CR MAC to the wireless physical layer. Such that the wireless physical layer (WirelessPhy) uses the coming information from channelindex _ in determining and using the most appropriate channel using the following predefined functions in $\mathrm{CRCN}$ simulator, which is the interface for channel decision in MAC:

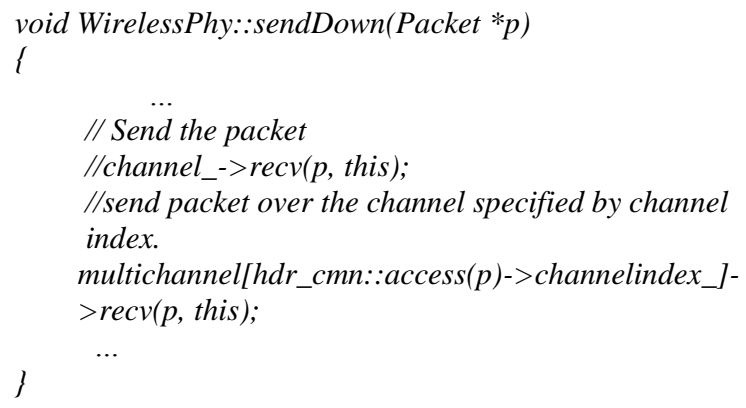

To know information about the positions of the nodes in a network to distribute channels among them, we use the function distance(MobileNode ${ }^{*} \mathrm{~m}$ ) that is defined in [mobilenode.cc]. 


\subsection{Integrating Power Control to MAC Layer in NS-2}

Power control is defined as varying the transmission power for reducing the energy consumption, limiting the interference, and improving the spatial channel reuse. In this section, we will illustrate the used TPC model in NS2 , where all modifications were carried in $\mathrm{C}++$ codes.

The first step that will be done in integrating TPC into NS-2 is to calculate the minimum transmit power required for the desired distance. Due to using the Tworay ground propagation model in the proposed protocol, this model will be used for calculating the minimum power which is represented as:

$$
P_{\min }=\frac{\left(\text { RxThreshold } \cdot d^{4} \cdot L\right)}{\left(G_{t} \cdot G_{r} \cdot h_{t}^{2} \cdot h_{r}^{2}\right)}
$$

Where RxThreshold is the receiver sensitivity (i.e., the minimum detected power by the receiver), $d$ is the distance between transmitter and intended receiver, $L$ is the system loss, $G_{t}$ and $G_{r}$ are the gain of the transmitting and receiving antennas, respectively, and $h_{t}$ and $h_{r}$ are the heights of the transmitting and receiving antennas, respectively.

The next step is to add the above equation to a specific interference tolerance in order to protect the communication from any unexpected interference, where all these modifications are made in wirelessphy.cc file, which is found in (mac) folder.

The most important thing done for TPC is to collect information about the intended receiver. Firstly, the RTS packet is sent from the transmitter with default maximum power to this receiver. Once the RTS packet reaches the interface of the receiver, sendUp(Packet * $p)$ function is called, which is the function of class WirelessPhy to calculate the received power of the packet and the minimum transmission power needed for successful transmission, where these information can be obtained by knowing the distance between the transmitter and receiver, and the propagation model, which are known to the receiver.

The first function that will be called by MAC when the RTS packet is received from PHY is the recv(Packet $* \mathrm{p}$, Handler $* \mathrm{~h}$ ) function that will call the two new added functions, which are Interference() and SetPInterference(p->txinfo_.RxPr) to notify of occurring interference and calculate the power of interference, respectively.

Indication of occurring interference can be determined using the $\operatorname{recv}()$ function, such that this function checks the MAC state. If the MAC state is idle (MAC_IDLE), the received packet ( $p$ ) is assigned to pktRx_ variable, and sets the receiving timer mhRecv_. Otherwise, if the node is already receiving another packet, MAC state is set to MAC_RECV which indicates occurrence of collision i.e. interference. For this, the above two interference functions will be called and the node will receive the packet with larger receiving power by at least CPThreshold.

After this, the receiving timer mhRecv _ calls the recvHandler, which then calls the recv_timer() function to check the type of the received packet [21]. Since the RTS packet is the packet that has been received, the recvRTS(Packet*) function will be called.

Once the recvRTS(Packet $*$ ) is called, the needed transmission power is calculated through invoking the new added function SetTxpower(p->txinfo_.RxPr, p$>$ txinfo_.Pmin, ETHER_ADDR(rf->rf_ta)), where this function uses the previous calculated receiving power and the minimum power.

Then, send CTS(ETHER_ADDR(rf->rf_ta), rf$>$ rf_duration) function is invoked to send the CTS packet. Delivering CTS packet follows the same steps as delivering RTS packet else in this case, once the recv_timer() function checks the received packet. Then, the recvCTS(Packet $*$ p) function is called.

Next, tx_resume() function is called that sends the DATA packet that is waiting. After this, once the DATA packet reaches the intended receiver, an acknowledgement packet would be transmitted with the new modified power level.

However, any future transmission between this pair of nodes, the source node transmits the RTS packet using the power that was used in last transmission for this intended receiver. Then, after receiving this RTS, the transmission power will be updated based on the environment.

\subsection{Exchange Information among Layers}

\section{CR Routing protocol}

Figure 4 shows the general structure for exchanging information mechanism between CR routing protocol and CR MAC to select and assign channels. Here, many modifications have been done to commensurate with the proposed protocol in this work.

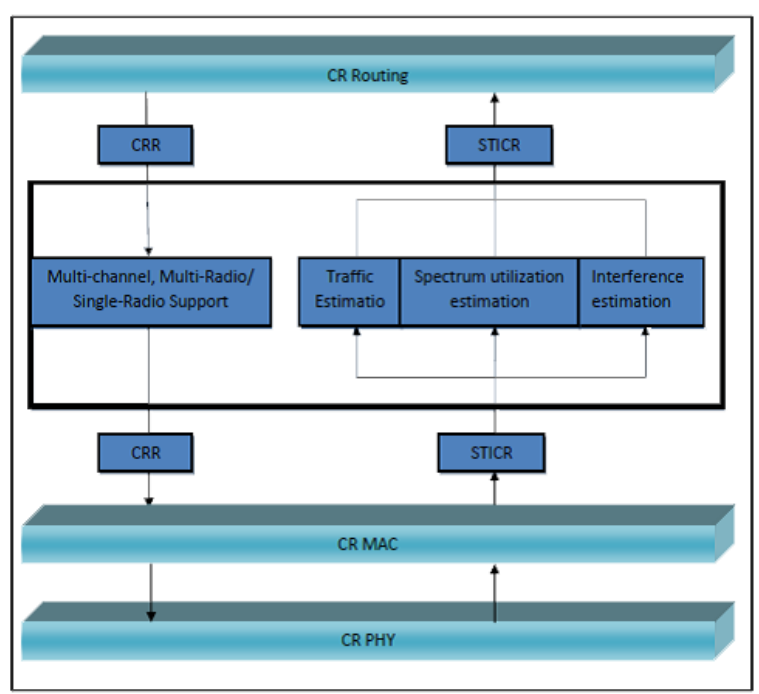

Figure 4. General design for CR routing mechanism [7]. 
Figure 4 shows the main functionalities supported by CRCN simulator for CR Routing in the darker color, where these functionalities are used to complete the jobs of this layer, which can be summarized as follows [7]:

- Channel decision, radio selection, and route selection decision (CRR): this job is summarized by defining the channel number, transmission power on the selected channel, and the path for packet forwarding, such that these decisions are sent to the lower layer through the functionalities supported by this simulator.

- Spectrum utilization information, traffic information and interference information from the channel and radio selected by CR Routing (STICR): this job is concluded by collecting information by the lower layer and then sending them to routing layer. This information mainly includes traffic, spectrum utilization, and interference information.

\section{CR MAC protocol}

Figure 5 shows the general design for contention based MAC protocol designed by CRCN simulator [7], where this layer has many functionalities that can be summarized as follows:

- Transmission power and channel selection (TC): after making decision by MAC, which includes the channel and transmission power selection, this simulator supports the functionality that sends this information to PHY through the multi-channel support functional block provided by this simulator.

- Traffic, interference, and communication over common channel information collection (TICC): this function has been added to make the physical layer capable of sending information about the traffic, interference, and communication information to the upper layer (MAC) through information block provided by this simulator.

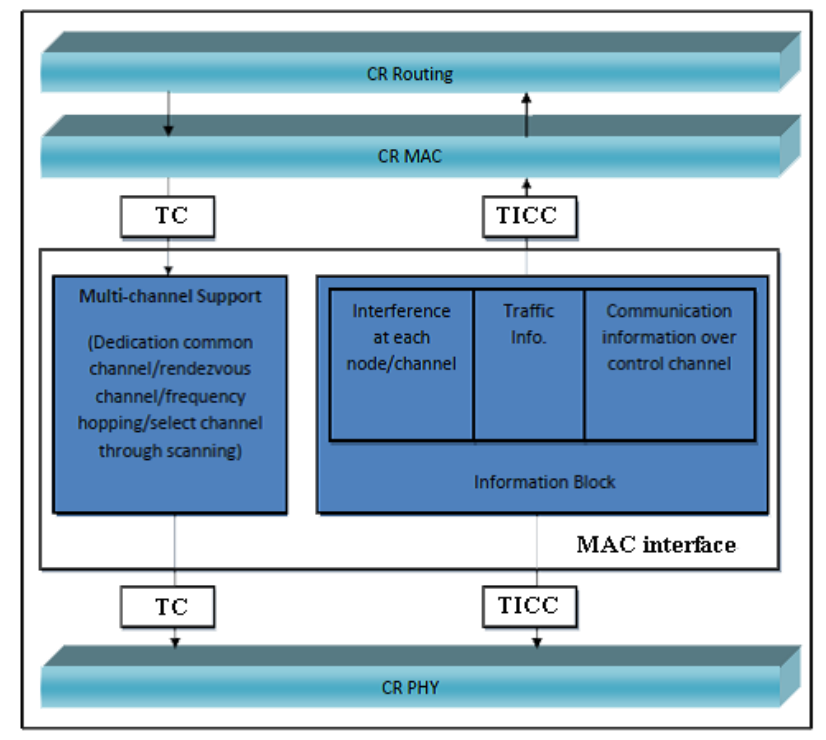

Figure 5. General design for CR MAC mechanism [7].

\section{Simulation Results}

In this work, NS-2 version 2.34 [24] was used for simulation. The simulation is used to demonstrate the network performance of the proposed protocol for both CRAHN without and with power control (PCCRMAC). All nodes are supported with single interface, and they are mobile with speed of $1(\mathrm{~m} / \mathrm{s})$. The nodes are located within an area of $30 \mathrm{~m} \times 30 \mathrm{~m}$, which is small and dense to show the effects of both CRAHN without power control and CRAHN with power control. The traffic type was CBR (constant bit rate) with packet rate equal to 500 packets per second, where each packet consists of $1 \mathrm{~KB}$.

\subsection{Throughput Performance for CRAHN}

Figure 6 shows the throughput performance for the proposed channel assignment that is proposed for CRAHN in order to reduce the costs using single radio. Many contributions have been made using multiple radios such as in $[7,10,13,21]$. For example, DOSS [13] was implemented using multiple transceivers and two separate bands to manage common information exchange and busy tones, such that by increasing the allocated spectrum, the probability of interfering with the primary users increases, where this is not used here. In the figure below the performance of using different number of channels has been studied. This section has studied the effect of increasing number of channels as well as increasing the number of communicating nodes. The number of channels was 2,3 , and 5 , whereas the number of nodes has varied from 2 to 14 . The simulation time was 200 seconds, where all the studies have been conducted at the worst conditions, such that all nodes begin their communication at the same time, and they continue their communication during simulation time.

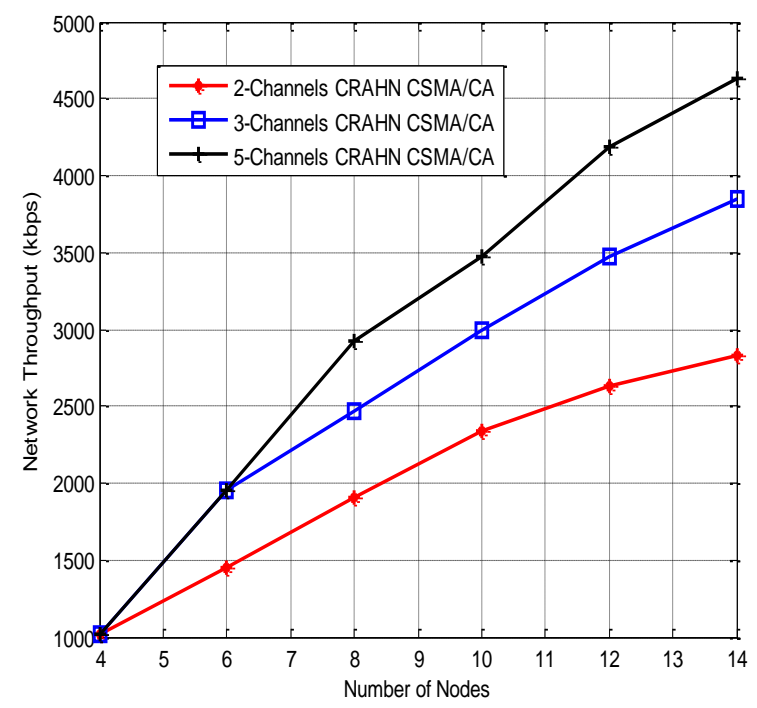

Figure 6. Throughput performance for CRAHN with CSMA/CA. 
As shown in the above figure, it can be noted that as the number of channels increases, the throughput increases. This is predictable because of decreasing the probability of interference as well as the collision. The second thing that can be seen is the effect of increasing the number of allowed nodes to communicate. It is obviously seen that increasing the number of nodes increases throughput.

\subsection{Transmission Power Control in CRAHNS}

\section{Throughput Improvement}

Most attempts to improve the performance of wireless networks through reducing interference have been addressed by introducing new channel assignments at MAC level in wireless networks such as in [4, 5,11], or using adaptive modulation and coding at PHY such as in [1, 6, 8]. In [11], Adaptive MAC (AMAC) proposed an algorithm for dynamic MAC protocol adaption in CRNs, such that each node has the ability of determining the appropriate MAC protocol from the available ones and switching between them if needed. In this algorithm, the used MAC protocols were CSMA and TDMA, and the result was an increase in throughput with $20 \%$ when compared with static CSMA and TDMA. Here in this proposed MAC protocol, a new channel assignment is introduced supported with power control algorithm for the goal of reducing interference. From the results below, it can be noted that a significant improvement has been achieved using the proposed algorithm, where the improvement varied from $26 \%$ to $67 \%$ for various numbers of channels and about $40 \%$ on average. However, the achieved enhancement may be due to the fact that TPC reduces the interference among links as well as increases the frequency spatial reuse. Figures 7, 8 , and 9 show the throughput performance for CRAHNs with and without the proposed protocol (PC-CRMAC) with various numbers of channels and nodes.

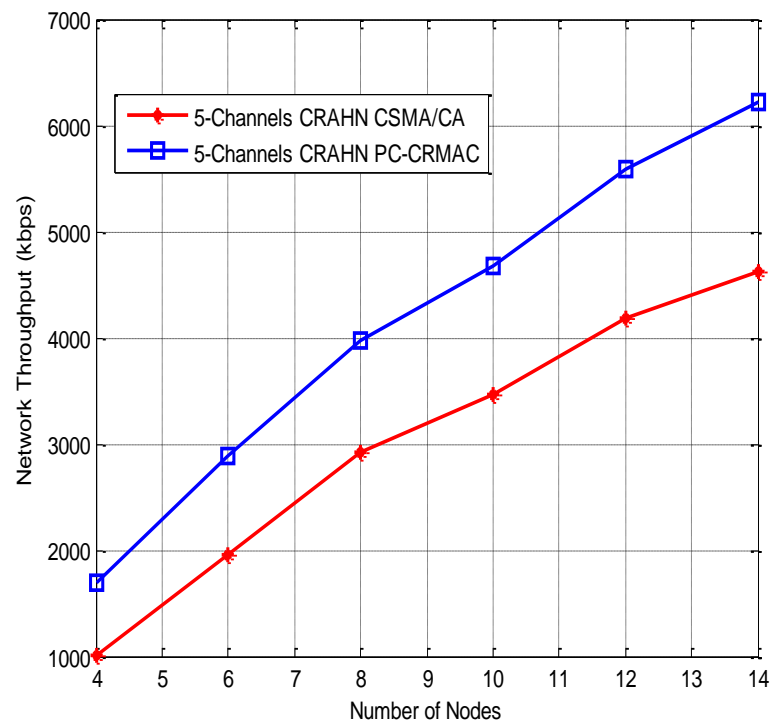

Figure 7. Average throughput with and without power control (5channels).

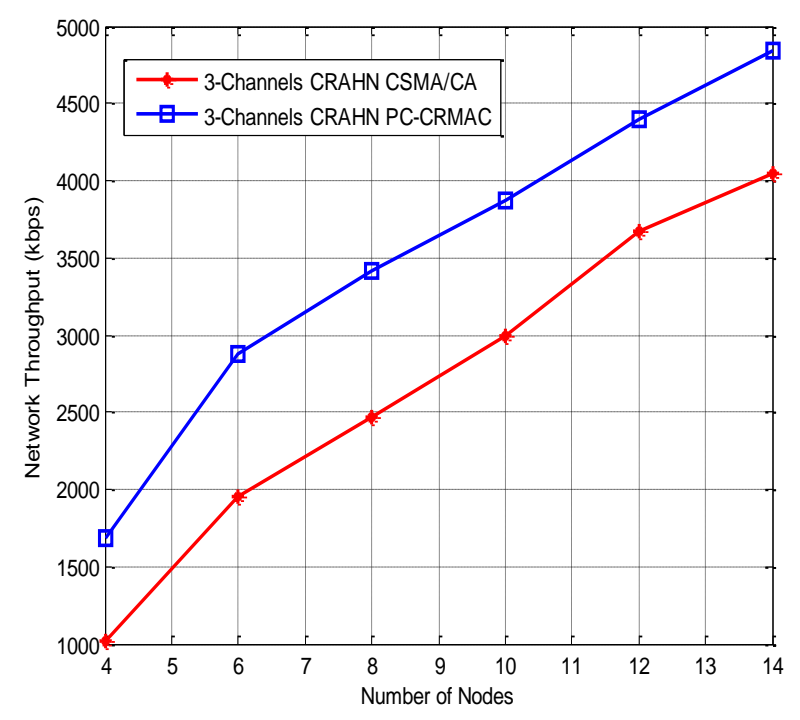

Figure 8. Average throughput with and without power control (3channels).

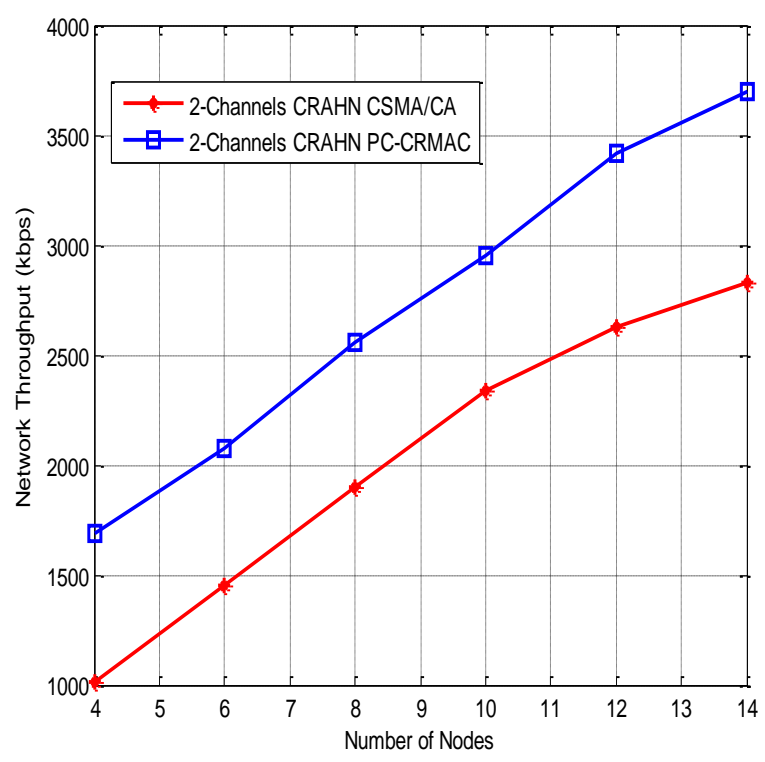

Figure 9. Average throughput with and without power control (2channels).

\section{Access Delay}

In this work, the proposed protocol PC-CRMAC was proposed mainly to enhance the network's throughput. From the above figures, it can be noticed that it is possible to increase the throughput and reduce the access delay using the proposed protocol. Increase in the throughput can be explained by reduction in access delay, where reduction in access delay allows users to reserve and leave the channel rapidly, which allows more communications. From Figure 10, as known the access delay increases as the number of communicating nodes increase, and this is verified using the simulator in this figure. 


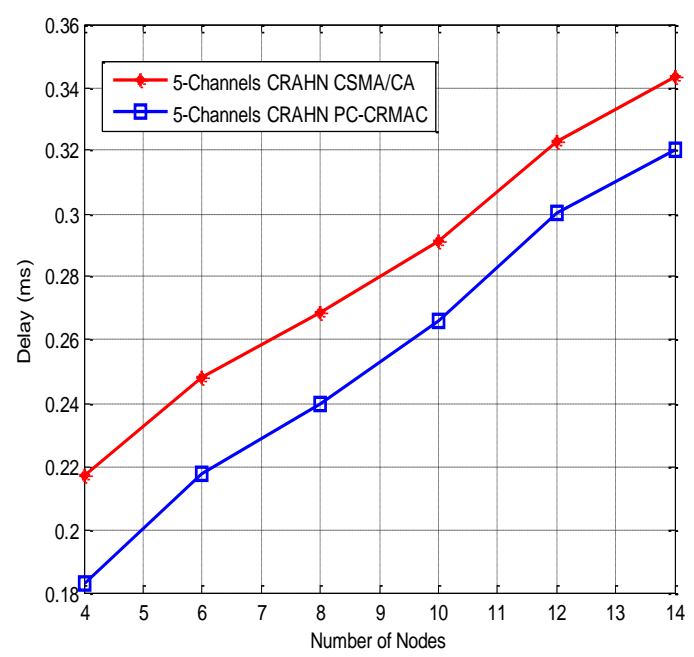

Figure 10. Access delay with and without power control (5channels).

\section{Conclusions}

In this work, the validation of integrating TPC to CRAHNs has been studied. Each node has the ability of determining the appropriate level of transmit power that ensures minimizing interference among users without affecting the reliability of communication. This has been seen on the throughput enhancement which is about $40 \%$ on average when using the proposed PC-CRMAC protocol as compared with using CRAHNs with CSMA/CA protocol.

\section{References}

[1] Aloqaily M., Al Ridhawi I., Bany Salameh H., and Jararweh Y., "Data and Service Management in Densely Crowded Environments: Challenges, Opportunities, and Recent Developments," IEEE Communications Magazine, vol. 57, no. 4, pp. 8187, 2019.

[2] Bany Salameh H., Kasasbeh H., and Harb B., "A Batch-Based MAC Design with Simultaneous Assignment Decisions for Improved Throughput in Guard-Band-Constrained Cognitive Networks," IEEE Transactions on Communications, vol. 64, no. 3, pp. 1143-1152, 2016.

[3] Bany Salameh H., Almajali S., Ayyash M., and Elgala H., "Spectrum Assignment in Cognitive Radio Networks for Internet-of-Things Delaysensitive Applications under Jamming Attacks," IEEE Internet of Things Journal, vol. 5, no. 3, pp. 1904-1913, 2018.

[4] Bany Salameh H. and AL-Quraan M., "Securing Delay-sensitive CR-IoT Networking under Jamming Attacks: Parallel Transmission and Batching Perspective," IEEE Internet of Things Journal, vol. 7, no. 8, pp. 4876-4883, 2020.

[5] Bany Salameh H., Krunz M., and Younis O., "Dynamic Spectrum Access Protocol Without Power Mask Constraints," in Proceedings of IEEE
INFOCOM 2009, Rio de Janeiro, pp. 2322-2330, 2009.

[6] Bany Salameh H., Almajali S., Ayyash M., Elgala H., "Security-Aware Channel Assignment in IotBased Cognitive Radio Networks For TimeCritical Applications," in Proceedings of $4^{\text {th }}$ International Conference on Software Defined Systems, Valencia, pp. 43-47, 2017.

[7] Cognitive Radio Cognitive Network Simulator, http://stuweb.ee.mtu.edu/ ljialian/, Last Visited 2019.

[8] Fantacci R., Marabissi D., Tarchi D., and Habib I., "Adaptive Modulation and Coding Techniques for OFDMA Systems," IEEE Transactions on Wireless Communication, vol. 8, no. 9, pp. 48764883, 2009.

[9] Gomez J. and Campbell A., "PARO: Supporting Dynamic Power Controlled Routing in Wireless Ad Hoc Networks," Wireless Networks, vol. 9, no. 5, pp. 443-460, 2003.

[10] Hong X., Chen Z., Wang C., Vorobyov S., and Thompson J., "Cognitive Radio Networks," IEEE Vehicular Technology Magazine, vol. 4, no. 4, pp. 76-84, 2009.

[11] Huang K. and Raychaudhuri D., "MAC Protocol Adaptation in Cognitive Radio Networks," in Proceedings of IEEE Wireless Communications and Networking Conference, Cancun, pp. 245250, 2011.

[12] Jondral F., "Software-Defined Radio: Basics and Evolution to Cognitive Radio," EURASIP Journal on Wireless Communications and Networking, vol. 5, no. 3, pp. 275-283, 2005.

[13] Jung E. and Vaidya N., "A Power Control MAC Protocol for Ad Hoc Networks," in Proceedings of $8^{\text {th }}$ ACM International Conference on Mobile Computing and Networking (MOBICOM), Atlanta, pp. 36-47, 2002.

[14] Khan A., Shoaib U., and Sarfraz M., "A comprehensive study of modern and high speed TCP-variant in linux kernel: TCP CUBIC," The International Arab Journal of Information Technology, vol. 16, no. 6, pp. 1028-1035, 2019.

[15] Monks J., Bharghavan V., and Hwu W., "A Power Controlled Multiple Access Protocol for Wireless Packet Networks," in Proceedings of IEEE INFOCOM-The Conference on Computer Communications, Anchorage, pp. 219-228, 2001.

[16] Muqattash A. and Krunz M., "POWMAC: A Single-Channel Power-Control Protocol For Throughput Enhancement In Wireless Ad Hoc Networks," IEEE Journal on Selected Areas in Communications, vol. 23, no. 5, pp. 1067-1084, 2005.

[17] Muqattash A. and Krunz M., "Power Controlled Dual Channel (PCDC) Medium Access Protocol for Wireless Ad Hoc Networks," in Proceedings 
of IEEE INFOCOM, San Francisco, pp. 470-480, 2003.

[18] Musa A., Bany Salameh H., Abu Sannad N., Halloush R., and Darabkh K., "Spectrum Management with Simultaneous Power-controlled Assignment Decisions in Cognitive Radio Networks," Concurrency and Computation: Practice and Experience, vol. 29, no. 3, pp. 1-12, 2019.

[19] Rabbachin A., Quek T., Shin H., and Win M., "Cognitive Network Interference," IEEE Journal on Selected Areas in Communications, vol. 29, no. 2, pp. 480-493, 2011.

[20] Rappaport T. and Milstein L., "Effects Of Radio Propagation Path Loss on DS-CDMA Cellular Frequency Reuse Efficiency For the Reverse Channel," IEEE Transactions on Vehicular Technology, vol. 41, no. 3, pp. 231-242, 1992.

[21] Robinson J., 802.11 MAC code in NS-2 (version2.28),

http://www.joshuarobinson.net/docs/802_11.html, Last Visited, 2019.

[22] Ruan L. and Lau V., "Decentralized Dynamic Hop Selection and Power Control in Cognitive MultiHop Relay Systems," IEEE Transactions on Wireless Communications, vol. 9, no. 10, pp. 30243030, 2010.

[23] Shorey R., Ananda A., Chan M., and Ooi W., Mobile, Wireless, and Sensor NetworksTechnology, Applications, and Future Directions, John Wiley and Sons, 2006.

[24] The network simulator. Retrieved May 05, 2006 from http://www.isi.edu/nsnam/ns/, Last Visited, 2019.

[25] Wu S., Tseng Y., Lin C., and Sheu J., "A MultiChannel MAC Protocol with Power Control for Multi-Hop Mobile Ad Hoc Networks," The Computer Journal, vol. 45, no. 1, pp. 101-110, 2002.

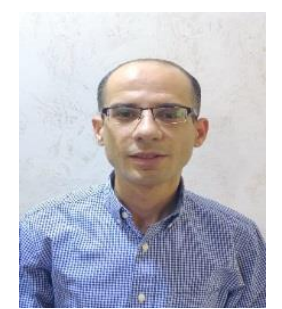

Ala'eddin Masadeh received the B.Sc. and the M.Sc. degrees in Electrical Engineering both from Jordan University of Science and Technology, Irbid, Jordan, in 2010 and 2013, respectively, and the Ph.D. degree in Electrical Engineering and Computer Engineering from Iowa State University, Ames, IA, USA, in 2019. Currently, he is an Assistant Professor in Electrical and Electronics Engineering Department, Al-Balqa Applied University, Jordan. His research interests include wireless networks, energy harvesting communications, reinforcement learning, machine learning, and artificial intelligence.

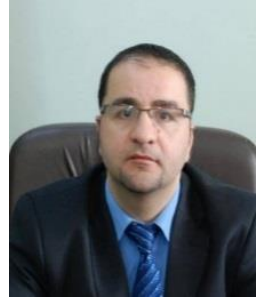

Haythem Bany Salameh (M'06SM'16) received the Ph.D. degree in Electrical and Computer Engineering from the University of Arizona, USA, in 2009. He is currently a Professor of Communications and Networking Engineering at Al Ain University, Al Ain, UAE (sabbatical leave from Yarmouk University (YU), Jordan). He also holds a visiting professor position at Staffordshire University, UK. He was a recipient of the Jordan Science Research Support Foundation (SRSF) Prestigious Award for Distinguished Research in ICT in 2015, the Best Researcher Award for Scientific Colleges in YU in 2016, and the SRSF Award for Creativity and Technological Scientific Innovation in 2017. From January 2011 to June 2014, he was the Director of the Academic Entrepreneurship Center of Excellence, YU. Prof. Bany Salameh was the Director of the Queen Rania Center for Jordanian Studies and Community Service from June 2014 to June 2018. His research interests include wireless networking, with emphasis on dynamic spectrum access, cognitive radio networking, Internet-of-Things, security, and distributed protocol design. Prof. Bany Salameh has served and continues to serve on the Technical Program Committee of many international conferences and serves as a Reviewer for many international conferences and journals. In the summer of 2008, he was a member of the RandD Long-Term Evolution Development Group QUALCOMM, Inc., San Diego, CA, USA. He is an IEEE Senior Member class of 2016.

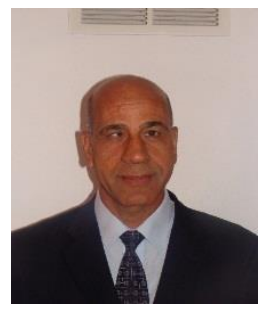

Ahmad Abu-El-Haija is the Director, National Tempus Office and Professor at Jordan University of Science and Technology (JUST). He was Vice President and Dean of Engineering at JUST. He conducted many consulting missions with World Bank, UNESCO, ITU, UN-ESCWA, ECDL Foundation, Islamic Development Bank, and others. He obtained scholarships and awards from the Fulbright (USA), Alexander-von-Humboldt and DAAD (Germany), Shoman Foundation (Arab region), United Nations. Dr. Abu-ElHaija obtained M.Sc. and Ph.D. from Stanford University, California, USA. He worked with IBM Research Center, New York (1977-1979). He published over 60 technical papers in leading scientific journals and proceedings of international conferences. 